\title{
CO-Dark Star Formation and Black Hole Activity in 3C 368 at $z=1.131$ : Coeval Growth of Stellar and Supermassive Black Hole Masses ${ }^{* \dagger}$
}

\author{
C. Lamarche $^{1}$, G. Stacey ${ }^{1}$, D. Brisbin ${ }^{2}$, C. Ferkinhoff ${ }^{3}$, S. Hailey-Dunsheath ${ }^{4}$, T. Nikola ${ }^{5}$, D. Riechers ${ }^{1}$, C. E. Sharon ${ }^{6}$, \\ H. Spoon ${ }^{5}$, and A. Vishwas ${ }^{1}$ \\ ${ }^{1}$ Department of Astronomy, Cornell University, Ithaca, NY 14853, USA; cj1272@ cornell.edu \\ ${ }^{2}$ Núcleo de Astronomía, Facultad de Ingeniería y Ciencias, Universidad Diego Portales, Avenida Ejército 441, 8370191 Santiago, Chile \\ ${ }^{3}$ Department of Physics, Winona State University, Winona, MN, 55987, USA \\ ${ }^{4}$ California Institute of Technology, Mail Code 301-17, 1200 East California Boulevard, Pasadena, CA 91125, USA \\ ${ }^{5}$ Cornell Center for Astrophysics and Planetary Science, Cornell University, Ithaca, NY 14853, USA \\ ${ }^{6}$ Department of Physics \& Astronomy, McMaster University, 1280 Main Street West, Hamilton, ON L85-4M1, Canada \\ Received 2016 November 1; revised 2016 December 20; accepted 2017 January 9; published 2017 February 13
}

\begin{abstract}
We present the detection of four far-infrared fine-structure oxygen lines, as well as strong upper limits for the $\mathrm{CO}(2-1)$ and [N II] $205 \mu \mathrm{m}$ lines, in 3C 368, a well-studied radio-loud galaxy at $z=1.131$. These new oxygen lines, taken in conjunction with previously observed neon and carbon fine-structure lines, suggest a powerful active galactic nucleus (AGN), accompanied by vigorous and extended star formation. A starburst dominated by $\mathrm{O} 8$ stars, with an age of $\sim 6.5 \mathrm{Myr}$, provides a good fit to the fine-structure line data. This estimated age of the starburst makes it nearly concurrent with the latest episode of AGN activity, suggesting a link between the growth of the supermassive black hole and stellar population in this source. We do not detect the $\mathrm{CO}(2-1)$ line, down to a level twelve times lower than the expected value for star-forming galaxies. This lack of $\mathrm{CO}$ line emission is consistent with recent star formation activity if the star-forming molecular gas has low metallicity, is highly fractionated (such that $\mathrm{CO}$ is photodissociated throughout much of the clouds), or is chemically very young (such that $\mathrm{CO}$ has not yet had time to form). It is also possible, although we argue it is unlikely, that the ensemble of fine-structure lines is emitted from the region heated by the AGN.
\end{abstract}

Key words: galaxies: evolution - galaxies: high-redshift - galaxies: ISM - galaxies: star formation - HII regions photon-dominated region (PDR)

\section{Introduction}

A major goal in the study of galaxy evolution is understanding the interplay between active galactic nuclei (AGNs) and star formation in their host galaxies. A correlation exists between the mass of central black holes that power AGN and the stellar bulge mass. This correlation suggests that the growth of black holes by accretion is linked to the growth of stellar mass by star formation (e.g., Ferrarese \& Merritt 2000). Gas accretion onto a central black hole can spur AGN activity, including winds and high-energy radiation, which may disrupt the ISM of the host galaxy, quenching star formation (e.g., Sanders et al. 1988). On the other hand, AGN activity through jets and winds, for example, can contribute additional pressure to the interstellar medium (ISM) of the host galaxy, leading to cloud collapse, and thereby bolstering star formation (both theoretically and observationally, e.g., Dey et al. 1997; Croft et al. 2006; Gaibler et al. 2012; Silk 2013). Here we investigate the relationship between star formation and AGN activity in the bright radio galaxy $3 \mathrm{C} 368$ at redshift $z=1.131$ (as determined by the [O II] $372.8 \mathrm{~nm}$ line, Meisenheimer \& Hippelein 1992). We observe 3C 368 as it was in the epoch of both peak star formation and AGN activity in the Universe.

3C 368 was discovered as part of the Third Cambridge Radio Catalog (Edge et al. 1959). Since then, it has become one of the

\footnotetext{
* Herschel is an ESA space observatory with science instruments provided by European-led Principal Investigator consortia and with important participation from NASA.

$\dagger$ Based on observations carried out with the IRAM Plateau de Bure Interferometer. IRAM is supported by INSU/CNRS (France), MPG (Germany) and IGN (Spain).
}

best-studied high-redshift, radio-loud, Fanaroff-Riley class II (FR-II) type galaxies, with observations spanning the electromagnetic spectrum from the radio (e.g., Best et al. 1998a) to the X-ray (e.g., Crawford \& Fabian 1995).

Not only does it exhibit considerable AGN activity, with radio emission extended over $73 \mathrm{kpc}$ (Best et al. 1998a), 3C 368 also has a substantial stellar mass $\left(\sim 3.6 \times 10^{11} M_{\odot}\right.$, Best et al. 1998b) and is undergoing a period of vigorous ( $350 M_{\odot} \mathrm{yr}^{-1}$, calculated by spectral energy distribution (SED) modeling in the far-infrared, Podigachoski et al. 2015) and extended (over $\sim 2-4 \mathrm{kpc}$, Stacey et al. 2010b) star formation. McCarthy et al. (1987) suggested a link between AGN activity and star formation in this source, in that the jets emanating from the AGN seem to be spurring star formation. To date, however, no $\mathrm{CO}$ emission, which traces the molecular gas necessary for star formation, has been detected in this source (e.g., Evans et al. 1996).

To further explore the properties of the stellar population in $3 \mathrm{C} 368$, including its age and spatial distribution, we observed the [O I] $63 \mu \mathrm{m}$ line, the [O III] 52 and $88 \mu \mathrm{m}$ lines, and the [O IV] $26 \mu \mathrm{m}$ line using the PACS spectrometer on the Herschel Space Observatory, the [N II] $205 \mu \mathrm{m}$ line with the Atacama Large Millimeter/submillimeter Array (ALMA), and the $\mathrm{CO}(2-1)$ line using both ALMA and the Plateau de Bure Interferometer (PdBI). The [OI] line arises from dense photodissociated gas on the surfaces of molecular clouds, and combined with our previous detection of the [C II] $158 \mu \mathrm{m}$ line with ZEUS on the Caltech Submillimeter Observatory (CSO) (Stacey et al. 2010b), traces the strength of the UV radiation field. The [O III] 52 and $88 \mu \mathrm{m}$ lines taken together allow us to 
estimate the density within $\mathrm{H}$ II regions, and the [O IV] $26 \mu \mathrm{m}$ line, with an ionization potential of $54.93 \mathrm{eV}$, traces the narrow line region (NLR) of the AGN.

Taken together with spectroscopic observations conducted with the Infrared Spectrograph (IRS) on board the Spitzer Space Telescope, including [Ne II] and [Ne III] lines in the midinfrared, these new data allow us to estimate the age of the starburst in 3C 368, furthering the theory of AGN-driven star formation in this powerful, yet enigmatic, galaxy.

We assume a flat $\Lambda$ CDM cosmology, with $\Omega_{M}=0.27$, $\Omega_{\Lambda}=0.73$, and $\mathrm{H}_{0}=71 \mathrm{~km} \mathrm{~s}^{-1} \mathrm{Mpc}^{-1}$, throughout this paper (Spergel et al. 2003).

\section{Observations}

\subsection{Herschel/PACS}

The oxygen fine-structure lines were observed in 3C 368 using the Photodetector Array Camera and Spectrometer(PACS) (Poglitsch et al. 2010) on board the Herschel Space Observatory (Pilbratt et al. 2010). The [O I] $63 \mu \mathrm{m}$ and [O III] $88 \mu \mathrm{m}$ lines were observed in line-scan chop-nod mode (Obs. ID: 1342243546), with a total observing time of $\sim 124$ minutes, before the [O III] $52 \mu \mathrm{m}$ and [O IV] $26 \mu \mathrm{m}$ lines were observed simultaneously in range-scan mode (Obs. ID: 1342243547), with an observing time of $\sim 38$ minutes. All observations took place on 2012 March 25.

These observations were reduced using the Herschel Interactive Processing Environment (HIPE, Version 13) (Ott 2010). For each of the oxygen lines, with the exception of the [O I] $63 \mu \mathrm{m}$ line, the central 9!" $4 \times 9$ "! 4 spatial pixel (spaxel) was used to generate the spectrum, and the results were point source corrected. For the [O I] $63 \mu \mathrm{m}$ line, the inner $3 \times 3$ spaxels of the integral field spectrometer were combined and point source corrected to create one spectrum. This step was taken in order to correct what appears to be pointing jitter with this observation, as indicated by the presence of line flux on multiple spaxels. Significant line flux was not seen to extend over multiple spaxels for any of the other lines observed by PACS.

These line fluxes are reported in Table 1 and the line spectra are shown in Figure 1.

\section{2. $P d B I$}

As a follow-up to our detection of the [C II] line, we attempted to detect the $\mathrm{CO}(2-1)$ rotational line with the Plateau de Bure Interferometer (PdBI) in 3C 368. The observations were carried out on 2010 May 31 and June 1, with five antennas in the $\mathrm{D}$ configuration, producing a synthesized beam of size 4". $69 \times 4$ !" 26 (FWHM). Total on-source integration was $5.25 \mathrm{hr}$, with the band-1 receivers tuned to the redshifted frequency of the line, $108.183 \mathrm{GHz}$. We used the WideX correlator, which provides an instantaneous $3.6 \mathrm{GHz}$ coverage in both polarizations. For this observation, we used MWC349 for absolute flux calibration. The data were calibrated and imaged using GILDAS, ${ }^{7}$ resulting in a $1 \sigma$ sensitivity of $279 \mu \mathrm{Jy}^{\text {beam }^{-1}}$ for this observation, calculated over an assumed line width of $500 \mathrm{~km} \mathrm{~s}^{-1}$; to match the width of the [C II] line. The line was not detected, with a $3 \sigma$ upper limit, assuming the source is small with respect to the $(39 \times 35 \mathrm{kpc})$ beam, of $0.42 \mathrm{Jy} \mathrm{km} \mathrm{s}^{-1}$, or equivalently $1.5 \times 10^{-21} \mathrm{~W} \mathrm{~m}^{-2}$.

\footnotetext{
7 https://www.iram.fr/IRAMFR/GILDAS
}

We detect the $1.3 \mathrm{~mm}$ rest-frame continuum in both the northern and southern lobes of 3C 368. We report these values using our subsequent more sensitive ALMA observations.

\subsection{ALMA}

Having made no detection with the PdBI, we next attempted to detect the $\mathrm{CO}(2-1)$ line in $3 \mathrm{C} 368$ with the Atacama Large Millimeter/submillimeter Array (ALMA). ${ }^{8}$ These band-3 observations were performed on 2015 January 31, with a synthesized beam size of 3 !" $12 \times 1$ "! 81 (FWHM). Our onsource integration time was $\sim 37$ minutes.

For these observations, which were conducted with a precipitable water vapor (PWV) measurement of $7.9 \pm 0.1 \mathrm{~mm}$, we used Titan as a flux calibrator, at a distance of 41.7 from the source, and J1751+0939 as both a phase and bandpass calibrator, located 3.6 from the source. Characteristic absolute flux calibrations with ALMA are accurate to $~ 10 \%$ (ALMA Technical Handbook). The data were reduced, imaged, and cleaned with the Common Astronomy Software Application (CASA), ${ }^{9}$ version 4.3.1.

The $1 \sigma$ sensitivity for these observations is $134 \mu \mathrm{Jy}_{\text {beam }^{-1}}$, over an assumed line width of $500 \mathrm{~km} \mathrm{~s}^{-1}$. Again the line is not detected. A $3 \sigma$ detection limit for a source enclosed by the $25.8 \times 15.0 \mathrm{kpc}$ beam yields an upper limit on the line intensity of $0.201 \mathrm{Jy} \mathrm{km} \mathrm{s}^{-1}$, or equivalently, $7.3 \times 10^{-22} \mathrm{~W} \mathrm{~m}^{-2}$.

This time, we detect the continuum in three components of 3C 368: the northern lobe at $673 \pm 39 \mu \mathrm{Jy}$, the southern lobe at $712 \pm 45 \mu \mathrm{Jy}$, and the core at $123 \pm 34 \mu \mathrm{Jy}$ (see Figure 2).

In addition to the $\mathrm{CO}(2-1)$ line, we attempted to detect the [N II] $205 \mu \mathrm{m}$ line with ALMA. These band-9 observations were carried out on 2014 February 21 and April 13, with a synthesized beam of size 0 ". $41 \times 0$ ". 29 (FWHM) and an onsource integration time of $\sim 54$ minutes.

For these observations, which were conducted with a PWV of $0.32 \pm 0.02 \mathrm{~mm}$, we used Titan as a flux calibrator, at a distance of $47^{\circ} .9$ from the source, J1751+0939 as a phase calibrator, located 3.6 from the source, and J1924-2914 as a bandpass calibrator, 44.77 from the source. Characteristic absolute flux calibrations for observations performed in cycle 1 with the ALMA band-9 receivers are accurate to $\sim 15 \%$ (ALMA Technical Handbook). The data were reduced, imaged, and cleaned with CASA, version 4.2.1.

We obtain a $1 \sigma$ sensitivity of $891 \mu \mathrm{Jy}_{\text {beam }}{ }^{-1}$, over an assumed line width of $500 \mathrm{~km} \mathrm{~s}^{-1}$. Presuming that the line source is extended over $\sim 2$ beams (as the continuum contours in Figure 2 suggest), the $3 \sigma$ upper limit is $3.78 \mathrm{Jy} \mathrm{km} \mathrm{s}^{-1}$ or $8.65 \times 10^{-20} \mathrm{Wm}^{-2}$.

We detect the rest-frame $205 \mu \mathrm{m}$ continuum, which traces thermal dust emission, from only the core component of 3C 368, with an integrated intensity of $5.6 \pm 1.3 \mathrm{mJy}$ (see Figure 2).

\subsection{Spitzer/IRS}

The [Ne II] $12.81 \mu \mathrm{m}$, [Ne III] $15.56 \mu \mathrm{m}$, and [Ne V] $14.32 \mu \mathrm{m}$ lines were obtained from the Cornell Atlas of Spitzer/IRS Sources (CASSIS, Version 6) (Lebouteiller et al. 2011), and were observed as part of a program to study multiple radio galaxies and quasars near the peak of cosmic star formation

\footnotetext{
8 The National Radio Astronomy Observatory is a facility of the National Science Foundation operated under cooperative agreement by Associated Universities, Inc.

9 https://casa.nrao.edu/
} 
Table 1

Spectral Lines Observed In 3C 368

\begin{tabular}{|c|c|c|c|c|c|c|c|c|c|c|c|}
\hline Line & $\begin{array}{l}{[\mathrm{Ne} \mathrm{II}]} \\
12.8 \mu \mathrm{m}\end{array}$ & $\begin{array}{c}{[\mathrm{Ne} \mathrm{V}]} \\
14.3 \mu \mathrm{m}\end{array}$ & $\begin{array}{l}{[\mathrm{Ne} \mathrm{III}]} \\
15.5 \mu \mathrm{m}\end{array}$ & $\begin{array}{l}{[\mathrm{O} \text { IV] }} \\
25.9 \mu \mathrm{m}\end{array}$ & $\begin{array}{l}{[\mathrm{Fe} \mathrm{II}]} \\
26.1 \mu \mathrm{m}\end{array}$ & $\begin{array}{l}{[\mathrm{O} \mathrm{III}]} \\
51.8 \mu \mathrm{m}\end{array}$ & $\begin{array}{l}{[\mathrm{O} \mathrm{I}]} \\
63.2 \mu \mathrm{m}\end{array}$ & $\begin{array}{c}{[\mathrm{O} \mathrm{III}]} \\
88.4 \mu \mathrm{m}\end{array}$ & $\begin{array}{c}{[\mathrm{C} \mathrm{II}]^{\mathrm{a}}} \\
158 \mu \mathrm{m}\end{array}$ & $\begin{array}{c}{[\mathrm{N} \text { II] }} \\
205 \mu \mathrm{m}\end{array}$ & $\begin{array}{c}\mathrm{CO}(2-1) \\
1.3 \mathrm{~mm}\end{array}$ \\
\hline Critical Density $\left(\mathrm{cm}^{-3}\right)$ & $7 \times 10^{5 \mathrm{~b}}$ & $3 \times 10^{4 \mathrm{~b}}$ & $3 \times 10^{5 b}$ & $1 \times 10^{4 \mathrm{~b}}$ & $1 \times 10^{4 \mathrm{~b}}$ & $3.6 \times 10^{3 \mathrm{c}}$ & $4.7 \times 10^{5 \mathrm{c}, \mathrm{d}}$ & $510^{\mathrm{c}}$ & $2.8 \times 10^{3 \mathrm{c}, \mathrm{d}}$ & $48^{\mathrm{c}}$ & $1.1 \times 10^{4 \mathrm{cc}, \mathrm{d}}$ \\
\hline Flux $\left(10^{-18} \mathrm{~W} \mathrm{~m}^{-2}\right)$ & $3.3 \pm 0.6$ & 1.5 & $9.1 \pm 0.6$ & 8.5 & 5.2 & $13.4^{\mathrm{e}}$ & 6.4 & 3.0 & 5.1 & $<0.0865$ & $<0.00073$ \\
\hline Significance $(\sigma)$ & $\ldots$ & 2.9 & $\ldots$ & 3.8 & 2.9 & 5.5 & 5.0 & 2.4 & 6.4 & $\ldots$ & $\ldots$ \\
\hline Line Center $\left(\mathrm{km} \mathrm{s}^{-1}\right)$ & $-40 \pm 210$ & $\ldots$ & $-40 \pm 100$ & $290 \pm 130$ & $220 \pm 60$ & $420 \pm 120$ & $240 \pm 40$ & $240^{\mathrm{f}}$ & -140 & $\ldots$ & $\ldots$ \\
\hline Line Width $\left(\mathrm{km} \mathrm{s}^{-1}\right)$ & $3100 \pm 700^{g}$ & $\ldots$ & $3000 \pm 200^{\mathrm{g}}$ & $510 \pm 330$ & $190 \pm 120$ & $760 \pm 290$ & $320 \pm 90$ & $320^{\mathrm{f}}$ & $\ldots$ & $\ldots$ & $\ldots$ \\
\hline
\end{tabular}

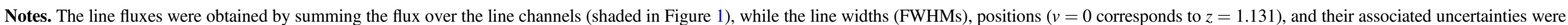
obtained from fitting Gaussian line profiles to the data. The fluxes for the higher signal-to-noise ratio neon lines were obtained from Gaussian fits to the line profiles.

$\omega \quad$ a Stacey et al. (2010b).

${ }^{\mathrm{b}}$ Cormier et al. (2012).

${ }^{\mathrm{c}}$ Carilli \& Walter (2013).

$\mathrm{d}$ These critical densities correspond to lines originating from neutral gas regions.

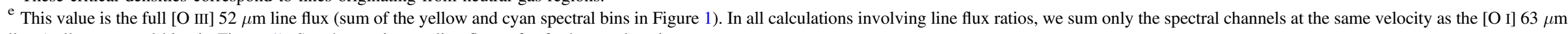
line (yellow spectral bins in Figure 1). See the section on line fluxes for further explanation.

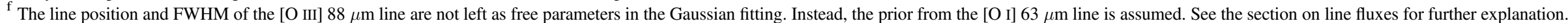

$\mathrm{g}$ The large FWHM of the fitted Ne lines is due to the resolution of the IRS in the LL1 observing mode and is not the physical width of these lines. 

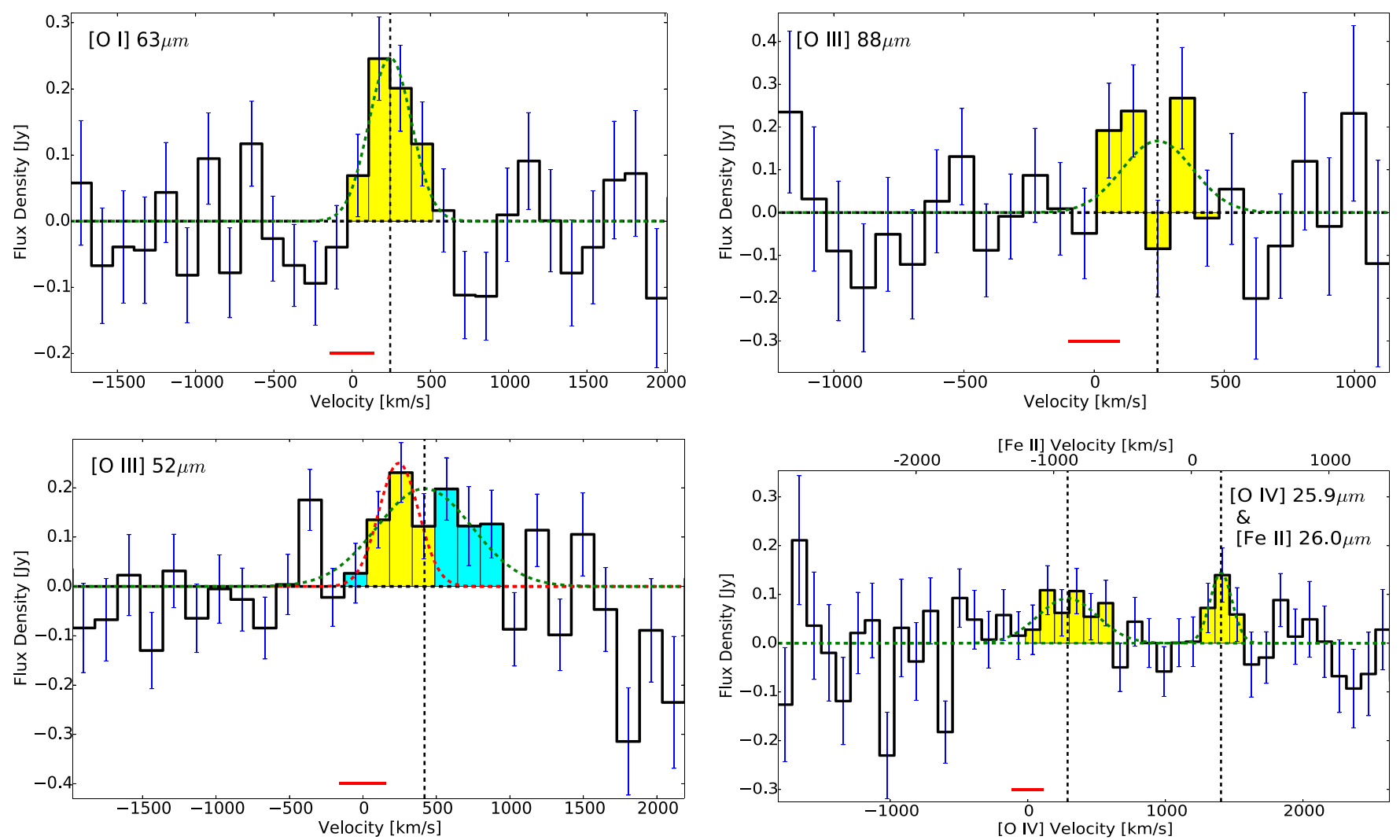

Figure 1. Spectral lines observed in 3C 368 with Herschel/PACS: (top left) [O I] $63 \mu \mathrm{m}$ line, spectral resolution $\sim 270 \mathrm{~km} \mathrm{~s}^{-1}$; (top right) [O III] $88 \mu \mathrm{m} \mathrm{line,} \mathrm{spectral}$ resolution $\sim 190 \mathrm{~km} \mathrm{~s}^{-1}$; (bottom left) [O III] $52 \mu \mathrm{m}$ line, spectral resolution $\sim 300 \mathrm{~km} \mathrm{~s}^{-1}$; (bottom right) [O IV] $25.9 \mu \mathrm{m}$ and [Fe II] $26.0 \mu \mathrm{m}$ lines, spectral resolution $\sim 210 \mathrm{~km} \mathrm{~s}^{-1}$. Gaussian fits to the line profiles are shown by the green dashed lines, with the central velocities indicated by vertical black dashed lines $(v=0$ corresponds to $z=1.131$ ). The blue error bars represent the $1 \sigma$ statistical uncertainty in the flux measurement in each channel, and the red bar below each spectrum indicates the velocity resolution of that spectrum. The flux in the [O III] $52 \mu \mathrm{m}$ line is calculated in two different ways, one including both the yellow and cyan spectral channels, and the other using only the yellow spectral channels (see section on line fluxes).

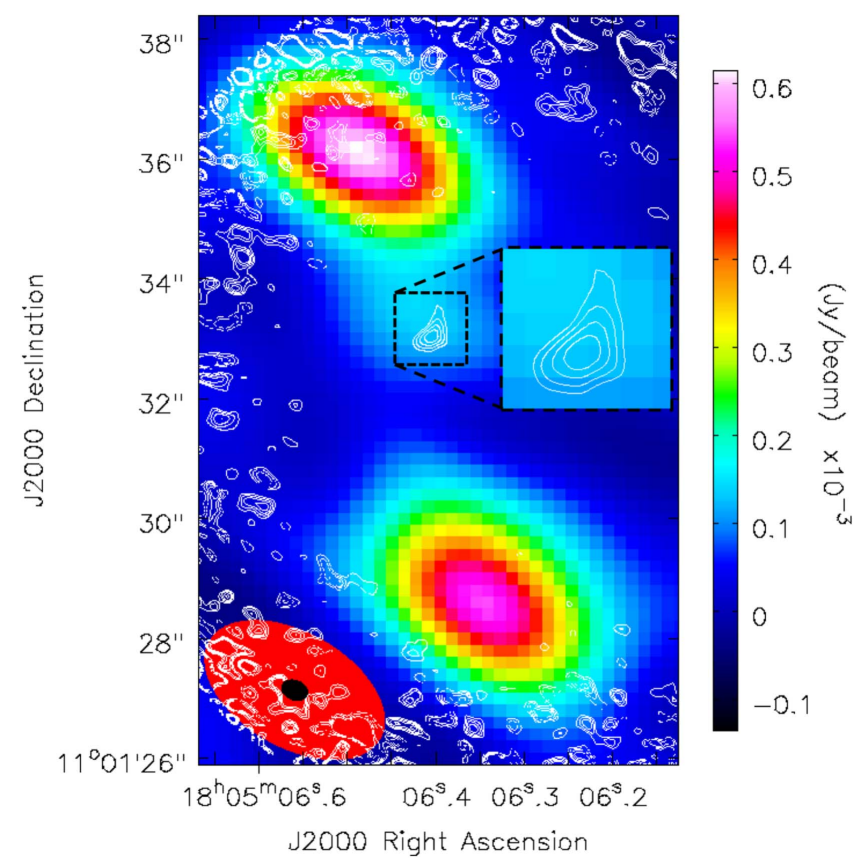

Figure 2. ALMA observations of the $1.3 \mathrm{~mm}$ (color map) and $205 \mu \mathrm{m}$ (contours) rest-frame continua in $3 \mathrm{C} 368$. The maps are primary-beam corrected. The contour levels are $\pm 3, \pm 4, \pm 5$, and $\pm 6 \sigma$ (negative contours are dashed, $1 \sigma=0.4 \mathrm{mJy}_{\text {beam }}{ }^{-1}$ ). The beams are shown at the bottom left corner of the image, with the large (red) and small (black) ellipses from the $1.3 \mathrm{~mm}$ and $205 \mu \mathrm{m}$ maps, respectively. The inset shows the $205 \mu \mathrm{m}$ restframe continuum from the core of $3 \mathrm{C} 368$. using the Spitzer Space Observatory (PI: Martin Haas, AORKEY $=22912000$ ). The observations of 3C 368 were carried out on 2008 May 1 with the InfraRed Spectrograph (IRS) (Houck et al. 2004) in low-resolution long-slit mode (LL1), covering a wavelength range of $19.5-38 \mu \mathrm{m}$. The total on-source integration time for these observations was $\sim 48$ minutes. The data were reduced and extracted using the CASSIS software, which employs an "optimal" extraction technique in order to produce a spectrum with the highest possible signal-to-noise ratio (Lebouteiller et al. 2015).

The line fluxes from the three neon lines are reported in Table 1 and the spectrum is plotted in Figure 3.

\section{Results and Discussion}

\subsection{Line Fluxes}

In order to determine the line fluxes for the fine-structure lines observed with Herschel/PACS, we sum the flux from the line channels (shaded in Figure 1), and propagate the statistical uncertainties per channel to calculate the signal-to-noise ratios. The channels associated with the spectral lines are determined by a Gaussian fit to the line profiles (see Table 1), with two exceptions.

First, the central line velocity and FWHM obtained by a Gaussian fit to the [O I] $63 \mu \mathrm{m}$ line, our most significant Herschel/PACS detection, are used to determine the line channels for the [O III] $88 \mu \mathrm{m}$ line. This is necessary because of the low signal-to-noise ratio in the [O III] $88 \mu \mathrm{m}$ line spectrum. 


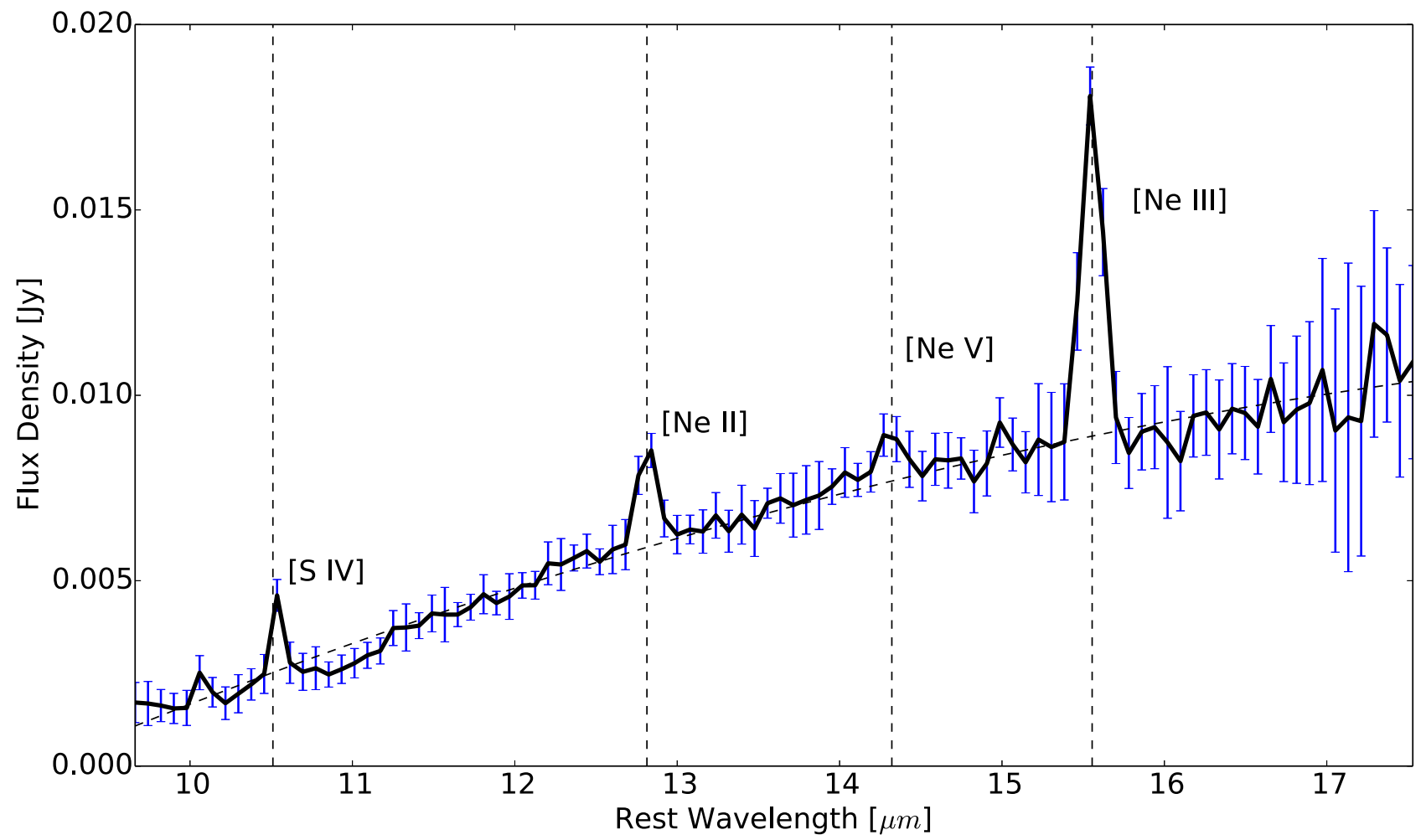

Figure 3. Spectral lines observed in 3C 368 with Spitzer/IRS. The positions of the various fine-structure lines are indicated by the vertical black dashed lines $(z=1.131)$. The blue error bars represent the $1 \sigma$ statistical uncertainty in the flux measurement in each channel. The spectral resolution ranges from $\sim 60-120$ over the wavelength range of this observation.

Second, a Gaussian fit to the [O III] $52 \mu \mathrm{m}$ line shows that it is considerably broader $\left(\Delta v \sim 760 \mathrm{~km} \mathrm{~s}^{-1}\right)$ than any of the other lines that we observed in 3C 368 (green dashed line, Figure 1). The reported flux for this line (see Table 1) is calculated by summing the flux from all channels under this best-fit Gaussian (shaded both yellow and cyan in Figure 1). Given that the signal-to-noise ratio per channel in the [O III] $52 \mu \mathrm{m}$ line spectrum is $\lesssim 2$ and that the fitted velocity width is so much larger than that of the [O I] $63 \mu \mathrm{m}$ line (our most significant oxygen line detection), we additionally calculate the flux by summing over only the channels coincident with our [O I] $63 \mu \mathrm{m}$ line (red dashed line and yellow shaded channels in Figure 1). We use this flux value, $6.8 \times 10^{-18} \mathrm{~W} \mathrm{~m}^{-2}$, in all calculations involving line ratios, since the flux excluded from this value originates within a different velocity component of 3C 368.

With the exception of the [O III] $52 \mu \mathrm{m}$ line, the central line velocities, $\sim+200-300 \mathrm{~km} \mathrm{~s}^{-1}$ (calculated using $z=1.131$ ), and velocity widths, $\sim 200-500 \mathrm{~km} \mathrm{~s}^{-1}$, calculated for the finestructure lines observed with Herschel/PACS are very similar.

The flux values, along with central line velocities and FWHMs, for the higher signal-to-noise ratio, [Ne II] $12.81 \mu \mathrm{m}$ and [Ne III] $15.56 \mu \mathrm{m}$ lines, observed with Spitzer/IRS were obtained from the Infrared Database of Extragalactic Observables from Spitzer (IDEOS) (Hernán-Caballero et al. 2016, H.W.W. Spoon et al. 2017, in preparation). The lower signalto-noise ratio flux, [Ne V] $14.32 \mu$ m line flux, was obtained by summing the line channels, exactly as done for the Herschel/ PACS spectra.

The central line velocities, determined by Gaussian fits to the [Ne II] $12.81 \mu \mathrm{m}$ and [Ne III] $15.56 \mu \mathrm{m}$ lines, overlap with those calculated for the oxygen lines observed with Herschel/ PACS within the $2 \sigma$ uncertainty level, indicating that these neon lines most likely originate within the same regions of 3C 368 as the oxygen lines.

\subsection{Neon Lines: Disentangling the AGN Contribution}

The mid-IR fine-structure lines in the Spitzer/IRS spectral regime that arise from the three ionization states of neon are excellent probes of the hardness of the ambient UV radiation fields in galaxies. It takes $21.56 \mathrm{eV}$ photons to form $\mathrm{Ne}^{+}$, $40.96 \mathrm{eV}$ photons to form $\mathrm{Ne}^{++}$, and $97.11 \mathrm{eV}$ photons to form $\mathrm{Ne}^{4+}$. Therefore, the $12.81 \mu \mathrm{m}$ [Ne II] line arises from $\mathrm{H}$ II regions formed by $\mathrm{O} / \mathrm{B}$ stars, the $15.56 \mu \mathrm{m}$ [Ne III] line arises from $\mathrm{H}$ II regions formed by $\mathrm{O}$ stars, and the $14.32 \mu \mathrm{m}[\mathrm{Ne} \mathrm{V}]$ line requires the very hard UV radiation fields found near AGN or planetary nebula created by hot white dwarfs. This series of lines is quite useful since extinction corrections between these lines are modest, and the lines have similar critical densities (Table 1). Furthermore, since neon is a noble gas, its gas phase abundance is not dependent on local conditions, since it is neither depleted onto grains nor incorporated into molecules.

[Ne V] line emission is known to be associated with planetary nebulae exposed to the hard UV radiation of very young white dwarfs (cf. Bernard Salas et al. 2001), but on galactic scales, detectable $[\mathrm{Ne} \mathrm{V}]$ is predominantly associated with the NLR of AGN. However, detectable [Ne III] emission can arise from both the NLR of AGN and from $\mathrm{H}$ II regions formed by hot $\mathrm{O}$ stars. Gorjian et al. (2007) studied the neon fine-structure line emission from a variety of galaxies, including $773 \mathrm{C}$ radio sources, and found that the amount of [Ne III] line emission from the NLR of 
AGN is directly proportional to the $[\mathrm{NeV}]$ line emission. Therefore, a simple scaling law, based on the observed [Ne V] line emission from a galaxy, can be applied to estimate the fraction of the observed [Ne III] line emission that arises from any central AGN. We use this scaling law to "correct" the [Ne III] line emission for the AGN contribution before applying any $\mathrm{H}$ II region models to the star-forming regions in 3 C 368 .

Applying the relation of Gorjian et al. (2007),

$$
\log \left(\frac{L_{\mathrm{Ne} \text { III }}}{10^{33} \mathrm{~W} \mathrm{sr}^{-1}}\right)=0.30+0.89 \log \left(\frac{L_{\mathrm{Ne} \mathrm{V}}}{10^{33} \mathrm{~W} \mathrm{sr}^{-1}}\right)
$$

to the observed luminosity of the [Ne V] $14.32 \mu \mathrm{m}$ line, we estimate that $20 \%$ of the observed [Ne III] emission originates from the AGN, leaving $80 \%$ from star-forming regions. This AGN-corrected $80 \%$ is the flux we use in our star formationdriven $\mathrm{H}$ II region models. We note that $3 \mathrm{C} 368$ is comparable to the higher luminosity sources used in the determination of this $[\mathrm{Ne} \mathrm{III]} /[\mathrm{Ne} \mathrm{V}]$ relation (Gorjian et al. 2007, Figure 1, right panel), where there are fewer sources and larger scatter in the trend, and so we also consider several limiting cases in the following analysis.

\subsection{H II Region Models}

Given the large number of fine-structure lines observed in 3C 368, we can determine several properties of the $\mathrm{H}$ II regions within this source.

The ground-state-term level populations within the $\mathrm{O}^{++}$ion are density sensitive, so that the [O III] $52 \mu \mathrm{m} /[\mathrm{O}$ III] $88 \mu \mathrm{m}$ line ratio yields the ionized gas density. We find a line ratio of 2.3, which indicates $\mathrm{H}$ II region gas densities of $\mathrm{n}_{\mathrm{e}} \sim 1000 \mathrm{~cm}^{-3}$ (we here use the collision strengths from Palay et al. 2012).

Then, using the models of Rubin (1985), we can combine the [O III], [Ne II], and AGN-corrected [Ne III] fine-structure lines to arrive at a consistent model for the $\mathrm{H}$ II regions in 3C 368 . We find that the fit favors regions of star formation that are heated by stars with effective temperatures of $\sim 37,000 \mathrm{~K}$ and with gas densities of $\sim 1000 \mathrm{~cm}^{-3}$. The density is consistent with our estimates from the [O III] 52/88 $\mu \mathrm{m}$ line ratio, and the ionization state is consistent with the neon line ratios, provided that neon is overabundant to oxygen in 3C 368 by a factor of three compared with either the "N" models, which have Orion Nebular abundances $\left(\mathrm{O} / \mathrm{H}=4.0 \times 10^{-4}\right.$ and $\left.\mathrm{N} / \mathrm{H}=4.5 \times 10^{-5}\right)$, or the "D" models, with all metals depleted by a factor of $\sqrt{10}$ from the " $\mathrm{N}$ " models.

This factor of three overabundance is consistent with the recent results of Rubin et al. (2016), who used Spitzer spectroscopy together with their ionization models and found neon-to-sulfur ratios $(\sim 12)$ for a wide variety of galaxies that were higher than previously expected. This suggests that the cannonical neon-to-sulfur ratio $(\sim 3.7)$ that was used in the $\mathrm{H} \mathrm{II}$ region models employed here is too low by a factor of about three. Since neon, sulfur, and oxygen are all primary elements, their abundances should scale together, and our neon overabundance when compared to oxygen is therefore consistent with this newly measured $\mathrm{Ne} / \mathrm{S}$ ratio.

Because the AGN contribution to the [Ne III] line is only $\sim 20 \%$, and because of the uncertainty in the line fluxes and the scatter in the $[\mathrm{Ne} \mathrm{III}] /[\mathrm{Ne} \mathrm{V}]$ correlation $(0.2 \mathrm{dex}$ in log space), we consider both the limiting case of no AGN contribution to the [Ne III] line and the case of the maximum allowed AGN contribution to the [Ne III] line $(\sim 40 \%)$. In all cases, we find that the models favor regions of star formation heated by stars with effective temperatures of $\sim 37,000 \mathrm{~K}$ and with gas densities of $\sim 1000 \mathrm{~cm}^{-3}$.

To retain the necessary effective stellar temperatures indicated by our modeling, at the observed far-IR luminosity in 3 C $368\left(2.0 \times 10^{12} L_{\odot}\right.$, Podigachoski et al. 2015), we require a starburst with the equivalent of $\sim 1.2 \times 10^{7} \mathrm{O} 8 \mathrm{~V}$ stars (Vacca et al. 1996). Such a starburst would have an age of $\sim 6.5 \mathrm{Myr}$ (Meynet \& Maeder 2003), as constrained by the stellar lifetimes of the most massive stars. Using the $73 \mathrm{kpc}$ radio source size of 3C 368 from Best et al. (1998a) and a range of jet propagation speeds from 0.03 to 0.3 c (King \& Pounds 2015), we estimate the duration of the latest episode of AGN activity to be between 0.4 and $4 \mathrm{Myr}$, comparable in time to the age of the starburst.

This concurrence lends further support to the possibility that we may be witnessing AGN-driven star formation in 3C 368, as has been suggested previously from the alignment of the radio and optical axes, which trace AGN jets and stars, respectively, seen in many of the 3CR galaxies, including 3C 368 (McCarthy et al. 1987). In addition to the optical continuum, Chambers et al. (1988) find the $2.2 \mu \mathrm{m}$ continuum, also associated with stellar emission, to be aligned with both of the other axes.

It has also been previously suggested that 3C 368 may be undergoing a major merger (Djorgovski et al. 1987), providing another possible mechanism for triggering both black hole accretion and star formation simultaneously. While we cannot rule out this possibility, the $[\mathrm{C} \mathrm{II}] / F_{\mathrm{FIR}}$ ratio of $0.5 \%$ in $3 \mathrm{C} 368$ (see Sections 3.5 and 3.6) is more than an order of magnitude higher than the typical values found in the local ultraluminous infrared galaxies (ULIRGs), which are predominantly powered by major-merger-driven starbursts (e.g., Luhman et al. 1998).

The physical parameters derived from our $\mathrm{H}$ II region modeling also explain our non-detection of the [N II] $205 \mu \mathrm{m}$ line. At such high effective temperatures, Rubin (1985) calculated that $\sim 86 \%$ of the nitrogen contained within the $\mathrm{H}$ II regions of $3 \mathrm{C} 368$ would be in the $\mathrm{N}^{++}$state. Unfortunately, the [N III] $57 \mu \mathrm{m}$ line is redshifted into an absorption band in the atmosphere for 3C 368 with $z=1.131(\sim 122 \mu \mathrm{m})$, making observations impossible from the ground, and even beyond the reach of airborne facilities. These observations will have to wait for the next generation of far-IR space telescopes, such as the Space Infrared Telescope for Cosmology and Astrophysics (SPICA) and the Origins Space Telescope (OST).

\subsection{Bounds on the N/O Ratio}

Following Ferkinhoff et al. (2010), we can estimate the minimum mass of oxygen in the $\mathrm{O}^{++}$state in 3C 368 from the luminosity in the oxygen fine-structure lines:

$$
M_{\min }\left(O^{++}\right)=\frac{F_{u l} 4 \pi D_{L}^{2} m_{O}}{\frac{g_{l}}{g_{t}} A_{u l} h \nu_{u l}},
$$

where $F_{u l}$ is the flux in the fine-structure line between the upper (u) state and the lower $(l)$ state, $D_{L}$ is the luminosity distance $(7.735 \mathrm{Gpc}), m_{O}$ is the mass of an oxygen atom, $g_{u}$ and $g_{l}$ are the statistical weights of the upper and lower states, respectively, $g_{t}$ is the partition function (the sum of the statistical weights of all relevant states available to the $\mathrm{O}^{++}$ion at $T=8,000 \mathrm{~K}), A_{u l}$ is the Einstein coefficient for the relevant 
transition $\left(2.6 \times 10^{-5} \mathrm{~s}^{-1}\right.$ for the [O III] $88 \mu \mathrm{m}$ line, Carilli \& Walter 2013), and $\nu_{u l}$ is the frequency of that transition. Using the [O III] $88 \mu \mathrm{m}$ line, we obtain a minimum $\mathrm{O}^{++}$mass of $4.4 \times 10^{6} M_{\odot}$.

Similarly, we can use our $3 \sigma$ upper limit for the [N II] $205 \mu \mathrm{m}$ line flux $\left(A=2.1 \times 10^{-6} \mathrm{~s}^{-1}\right.$, Carilli \& Walter 2013) to place a bound on the mass of $\mathrm{N}^{+}$in $3 \mathrm{C} 368$ :

$$
M_{\min }\left(N^{+}\right)<\frac{F_{u l} 4 \pi D_{L}^{2} m_{N}}{\frac{g_{l}}{g_{t}} A_{u l} h \nu_{u l}} .
$$

We obtain a limit of $<3.2 \times 10^{6} M_{\odot}$.

We use the models of Rubin (1985) to calculate the total mass in oxygen and nitrogen by scaling from the fraction in the ionization state that we observe back to the total abundance of the element in question.

With $\sim 84 \%$ of the oxygen in the $\mathrm{O}^{++}$state, and $\sim 14 \%$ of the nitrogen in the $\mathrm{N}^{+}$state, within the $\mathrm{H}$ II regions of $3 \mathrm{C} 368$, we calculate masses of $5.3 \times 10^{6} M_{\odot}$ and $<2.3 \times 10^{7} M_{\odot}$, for oxygen and nitrogen respectively.

Using these values, we find an upper limit for the $\mathrm{N} / \mathrm{O}$ ratio in 3C 368 of 5.0. Asplund et al. (2009) reported a solar N/O abundance ratio of 0.126 . While the result we obtain is consistent with the solar $\mathrm{N} / \mathrm{O}$ ratio, it also allows for enhanced nitrogen relative to oxygen.

We are continuing our campaign to detect the [N II] $205 \mu \mathrm{m}$ line in 3C 368 and were recently granted ALMA time to push our sensitivity deeper in this source. These new observations will effectively double our on-source integration time, which will improve our sensitivity.

\subsection{Photodissociation Regions}

In addition to the lines associated with $\mathrm{H}$ II regions, we have also detected the [O I] $63 \mu \mathrm{m}$ line associated with photodissociation regions (PDRs). [O I] and [C II] are the two dominant coolants of PDRs, so that the sum of the line fluxes divided by the far-IR flux (which tracks the impinging FUV (6-13.6 eV) flux) tracks the efficiency of photoelectric heating. Parameterizing the FUV in terms of the local (Habing) interstellar radiation field, the photoelectric heating is sensitive to the ratio of FUV field strength to gas density, $G_{0} / n$. With different critical densities the line ratio constrains the PDR gas density, so that the line intensities and their ratio independently constrain $G_{0}$ and $n$.

We use our previous detection of [C II] (Stacey et al. 2010b) together with the PDR Toolbox (Kaufman et al. 2006) for our analysis. This software fits input line flux ratios to a bank of line ratios calculated using a radiative transfer code that assumes a plane-parallel geometry for the gas.

Before using this software, we correct for the different optical depths of the lines used in modeling the PDRs. The [O I] $63 \mu \mathrm{m}$ line is likely to be optically thick for PDRs with $A_{V}$ on the order of a few (e.g., Stacey et al. 1983; Tielens \& Hollenbach 1985). We therefore multiply the [O I] $63 \mu \mathrm{m}$ line flux by a factor of two to account for its opacity (as in Stacey et al. 2010a), taking the uncertainty in this correction to be equal to the correction we applied.

Owing to the lower abundance of $\mathrm{C}^{+}$and the greater populations in its excited levels, the [C II] line is expected to have significantly smaller optical depth than the [O I] line. Observations of the hyperfine structure lines of ${ }^{13} \mathrm{C}^{+}$indicate optical depths lower than or on the order of $0.5-1.5$ for galactic
Table 2

PDR Modeling Line Corrections

\begin{tabular}{lccr}
\hline \hline Line & {$[\mathrm{O} \mathrm{I}]$} & {$[\mathrm{C} \text { II }]^{\mathrm{a}}$} & $F_{\mathrm{FIR}}{ }^{\mathrm{b}}$ \\
& $63.2 \mu \mathrm{m}$ & $158 \mu \mathrm{m}$ & \\
\hline Uncorrected Flux $\left(10^{-18} \mathrm{~W} \mathrm{~m}^{-2}\right)$ & 6.4 & 5.1 & 1075.0 \\
Corrected Flux $\left(10^{-18} \mathrm{~W} \mathrm{~m}^{-2}\right)$ & 12.8 & 5.1 & 1075.0 \\
Uncertainty $\left(10^{-18} \mathrm{~W} \mathrm{~m}^{-2}\right)$ & $6.4^{\mathrm{c}}$ & 1.5 & 107.5 \\
\hline
\end{tabular}

Notes.

${ }^{\text {a }}$ Stacey et al. (2010b).

b Podigachoski et al. (2015).

${ }^{\mathrm{c}}$ We take the uncertainty in the [O I] $63 \mu \mathrm{m}$ line to be equal to the opacity correction we applied.

PDRs (see Boreiko et al. 1988; Stacey et al. 1991; Ossenkopf et al. 2013). PDR models indicate optical depths of the same order (Kaufman et al. 1999). Therefore, we make no correction to the [C II] $158 \mu \mathrm{m}$ line for opacity effects.

We perform an additional correction to remove any contributions to the [C II] $158 \mu \mathrm{m}$ line emission originating from $\mathrm{H}$ II regions, which we subtract before performing our PDR analysis. With an ionizing energy of only $11.26 \mathrm{eV}$, ionized carbon can be found in the neutral hydrogen phases of the ISM (13.6 eV ionizing energy) and also in the ionized gas phase where species like ionized nitrogen $(14.5 \mathrm{eV})$ exclusively reside (Oberst et al. 2006). Since the [N II] $205 \mu \mathrm{m}$ and [C II] $158 \mu \mathrm{m}$ lines have very similar critical densities in ionized gas regions, $48 \mathrm{~cm}^{-3}$ and $50 \mathrm{~cm}^{-3}$, respectively, their ratio can be used to calculate the percentage of $\mathrm{C}^{+}$coming from $\mathrm{H}$ II regions (Oberst et al. 2006). We calculate a flux ratio of [C II] $158 \mu \mathrm{m} /[\mathrm{N} \mathrm{II}] 205 \mu \mathrm{m}>60$. Oberst et al. (2006) find that if all of the $\mathrm{C}^{+}$observed were to come from $\mathrm{H}$ II regions, this ratio would vary from 3.1 for a low-density gas to 4.3 for a highdensity gas, so that $<7 \%$ of the [C II] $158 \mu$ m line flux in 3C 368 comes from within H II regions. Since this contribution is very small and within the uncertainties, we make no correction to the [C II] $158 \mu \mathrm{m}$ line flux for our PDR analysis.

Finally, we correct the observed FIR luminosity to include only the component produced by the UV flux from young stars that has been reprocessed by the dust into the FIR. We adopt the fit from Podigachoski et al. (2015), who modeled the SED of 3C 368 with three components: one for the AGN-fueled warm dust component (using a library of torus models from Hönig \& Kishimoto 2010), one blackbody for the optical/NIR stellar emission, and one graybody in the FIR/submillimeter region with the dust emissivity index $(\beta)$ as a free parameter. We use the luminosity in this last component for our PDR analysis. Podigachoski et al. (2015) also used this FIR luminosity to calculate a star formation rate in 3C 368, obtaining a value of $350 M_{\odot} \mathrm{yr}^{-1}$.

Inserting these three observations (see Table 2), the corrected [C II] $158 \mu \mathrm{m}$ and [O I] $63 \mu \mathrm{m}$ line intensities, and the fitted FIR luminosity into the PDR Toolbox software (Kaufman et al. 2006), we obtain best fits for PDRs with $G_{0} \sim 280$ and $\mathrm{n} \sim 7500 \mathrm{~cm}^{-3}$ (see Figure 4). Using the scaling relations found in Wolfire et al. (1990) and adopting an FIR luminosity of $2.0 \times 10^{12} L_{\odot}($ Podigachoski et al. 2015), we calculate that these PDRs are extended over $1.9-4.8 \mathrm{kpc}$, which is consistent with our CASA 2D Gaussian fit to the source from the restframe $205 \mu \mathrm{m}$ continuum map $(\sim 1.0 \times 5.9 \mathrm{kpc}$, see Figure 2$)$.

One potential caveat for our PDR modeling results is that the observed $[\mathrm{C} \mathrm{II}]$ and $[\mathrm{O} \mathrm{I}]$ lines may originate within the X-ray 


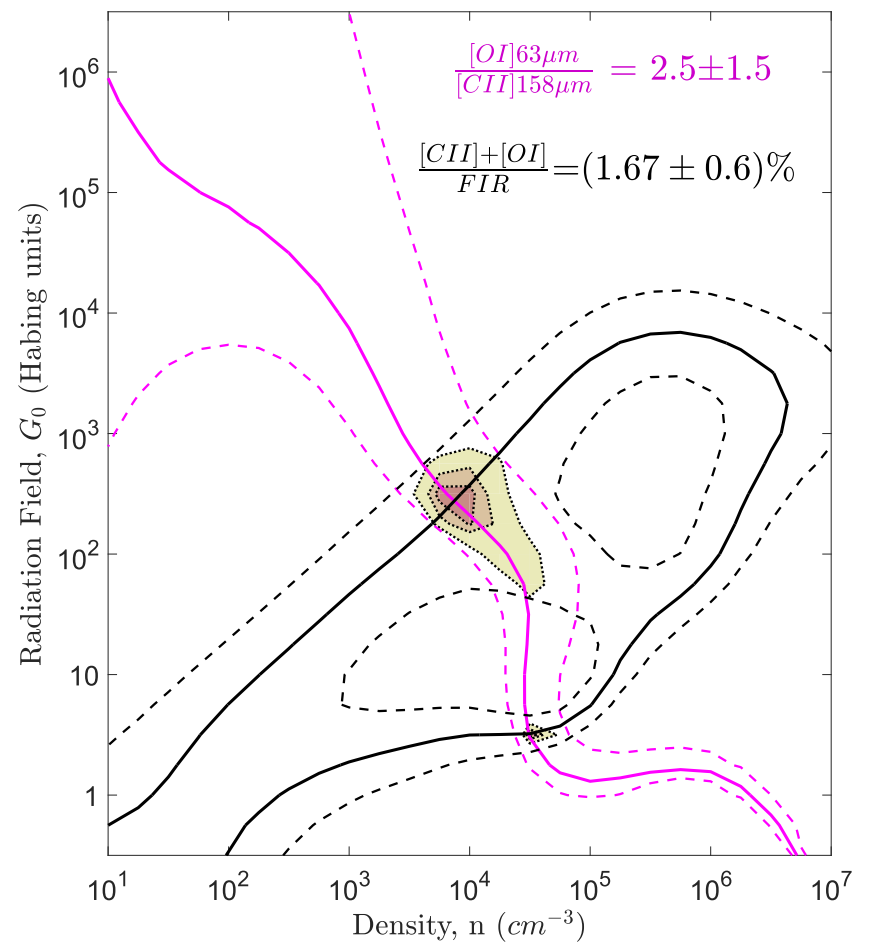

Figure 4. A plot of the best-fit PDR parameters in 3C 368, using the PDR Toolbox (Kaufman et al. 2006). The black (magenta) solid line traces the values of the radiation field $\left(G_{0}\right)$ and density $(n)$ allowed by the $[\mathrm{C} \mathrm{II}]+[\mathrm{O} \mathrm{I}] /$ FIR ([O I] /[C II]) ratio and is bounded by the uncertainty in the ratio (dashed lines). The three filled contours represent $\chi^{2}$ fitting values of $0.25,0.5$, and 1.0 The fit favors PDRs with $G_{0} \sim 280$ and $\mathrm{n} \sim 7500 \mathrm{~cm}^{-3}$. The low $G_{0}$ solution is disregarded because it would result in an emitting region with an unphysically large size.

dominated region (XDR) surrounding the AGN of 3C 368 (e.g., Meijerink et al. 2007), with the [O III] lines coming from the closer-in NLR (e.g., Ferkinhoff et al. 2010). Because we can form a consistent picture of star formation in 3C 368 using our observations and because optical fine-structure lines have also been observed to be extended over several arcseconds around the central source (e.g., Hammer et al. 1991; Meisenheimer \& Hippelein 1992; Jackson \& Rawlings 1997; Best et al. 2000), the theory of large-scale star formation in 3C 368 seems more plausible.

This theory can be tested by spatially resolving the $[\mathrm{C} \mathrm{II}]$ line emission in 3C 368. Extended [C II] emission may confirm the existence of large-scale star-forming regions, while confined emission may suggest an AGN origin. We have been awarded ALMA time to carry out these very observations (see the section on future observations).

\subsection{Where is the CO?}

While the fine-structure lines from PDRs allow us to model these regions in $3 \mathrm{C} 368$, the complete picture of star formation can only come from including molecular gas observations as well. We have attempted to observe the $\mathrm{CO}(2-1)$ transition with both ALMA and PdBI, and like others in the past (e.g., Evans et al. 1996), we did not detect any emission. Our ALMA observations probe down to a sensitivity of $134 \mu \mathrm{Jy}$ beam $^{-1}$ over an assumed line width of $500 \mathrm{~km} \mathrm{~s}^{-1}$.

Assuming a ratio of $[\mathrm{C} \mathrm{II}]$ to $\mathrm{CO}(1-0)$ of $\sim 4100$, which is typical of normal metallicity star-forming regions in the Milky Way and starburst galaxies (Stacey et al. 1991), and a CO(2-1) to $\mathrm{CO}(1-0)$ flux ratio of 7.2 (90\% of the high-temperature thermalized value), we would expect a [C II] to $\mathrm{CO}(2-1)$ flux ratio of $\sim 570$ in $3 \mathrm{C} 368$. The $3 \sigma \mathrm{CO}$ upper limit from our ALMA observations, however, yields a limit of $[\mathrm{C} \mathrm{II}] / \mathrm{CO}$ $(2-1)>7000$, which is more than 12 times the expected value.

Following Scoville et al. (2016), we can estimate the total molecular gas mass in $3 \mathrm{C} 368$ using the $850 \mu \mathrm{m}$ SCUBA observations from Archibald et al. (2001). Assuming a dust temperature of $35 \mathrm{~K}$, consistent with the SED fit from Podigachoski et al. (2015), we obtain a value of $1.57 \times 10^{11} M_{\odot}$. Then, using our upper limit for the $\mathrm{CO}(2-1)$ line and assuming that the gas is fully thermalized, we obtain a $3 \sigma$ limit for $L_{\mathrm{CO}}^{\prime}<3.45 \times 10^{9} \mathrm{~K} \mathrm{~km} \mathrm{~s}^{-1} \mathrm{pc}^{2}$. Using these values, we estimate an $\alpha_{\mathrm{CO}}>45.5 M_{\odot}\left(\mathrm{K} \mathrm{km} \mathrm{s}^{-1} \mathrm{pc}^{2}\right)^{-1}$ in 3C 368. This $\alpha_{\mathrm{CO}}$ value is an order of magnitude higher than the typical range of values quoted in the literature, $\sim 0.8-4.3$ (e.g., Solomon \& Vanden Bout 2005; Bolatto et al. 2013).

Previous attempts to detect molecular gas using the $\mathrm{CO}(1-0)$ line in high-redshift radio galaxies have been largely unsuccessful (e.g., Emonts et al. 2014). Our limit of $L_{\text {CO }}^{\prime}<3.45 \times 10^{9} \mathrm{~K} \mathrm{~km} \mathrm{~s}^{-1} \mathrm{pc}^{2}$ is a factor of $\sim 6.4$ deeper than the lowest limit reported in Emonts et al. (2014) $\left(L_{\mathrm{CO}}^{\prime}<2.2 \times 10^{10} \mathrm{~K} \mathrm{~km} \mathrm{~s}^{-1} \mathrm{pc}^{2}\right)$.

Comparing the observed $[\mathrm{C} \mathrm{II}] / F_{\mathrm{FIR}}$ and upper limit $\mathrm{CO}$ $(1-0) / F_{\text {FIR }}$ ratios for $3 \mathrm{C} 368$ to those of other sources (HaileyDunsheath et al. 2010, Figure 3), we find that 3C 368 has a higher $\left[\mathrm{C}\right.$ II] $/ F_{\text {FIR }}$ ratio $(\sim 0.5 \%)$ than either the starburst nuclei or galactic star-forming regions, and deficient $\mathrm{CO}$ for a source with this [C II] flux. There are several factors that might explain this anomalously high [C II] $/ \mathrm{CO}(2-1)$ line ratio, including the metallicity, age, and degree of fractionation of the molecular clouds in 3C 368 .

Moving toward the core of a molecular cloud from its surface, a transition is made from $\mathrm{C}^{+}$to $\mathrm{CO}$ as carbon-ionizing and CO-dissociating photons are extincted by dust. This transition occurs at $A_{V} \sim 3$ (Hollenbach \& Tielens 1999). If the dust-to-gas ratio follows metallicity, then it follows that the penetration depth of these carbon-ionizing photons can be very large in a low-metallicity ISM, making the $\mathrm{CO}$ core relatively small compared to the $\mathrm{C}^{+}$envelope, and leading to high [C II]/CO ratios (e.g., Maloney \& Black 1988; Stacey et al. 1991). While the CO-emitting core of a molecular cloud retreats as the metallicity decreases, the size of the molecular region, defined by the transition from $\mathrm{H}$ to $\mathrm{H}_{2}$, does not. This transition from $\mathrm{H}$ to $\mathrm{H}_{2}$ is set by the self-shielding of $\mathrm{H}_{2}$ molecules and is therefore metallicity independent. $\mathrm{CO}$ would therefore be a poor tracer of the total molecular gas in lowmetallicity molecular clouds, which could still be present in the near absence of CO (e.g., Stacey et al. 1991; Poglitsch et al. 1995; Madden et al. 1997).

However, the low-metallicity case is unlikely for 3C 368, which has a stellar mass of $\sim 3.6 \times 10^{11} M_{\odot}$ (Best et al. 1998b). At the current star formation rate, it would take $\sim 10^{9}$ years to accumulate such a stellar population. Assuming that each star formation episode lasts $\sim 10^{7}-10^{8}$ years, 3C 368 would have been through $\sim 10-100$ cycles of star formation, making a lowmetallicity ISM seem improbable. Additionally, 3C 368 lies on the galaxy main sequence for a source at redshift 1.131 (using the definition of Genzel et al. 2015), furthering the idea that a low-metallicity ISM is not responsible for the lack of CO in this case. 
A different scenario that might be used to explain the lack of $\mathrm{CO}$ is that the ISM in 3C 368 may be highly fractionated into small cloudlets. Such a fractioned ISM would have modest extinction to the core of individual cloudlets, allowing for considerable $\mathrm{C}^{+}$emission with relatively little $\mathrm{CO}$ emission.

Since the upper limit from our CO observation shows that the line is suppressed from the expected value by more than 12 times, the CO-emitting cores of the molecular clouds in 3C 368 must correspondingly be more than $\sqrt{12} \times$ smaller in radius than the entire clouds. Taking $A_{V} \sim 3$, or a corresponding column density of $6 \times 10^{21} \mathrm{~cm}^{-2}$, as the CO depth, and our fitted PDR density of $\sim 7500 \mathrm{~cm}^{-3}$, we calculate that the clouds in 3 C 368 must have size $\sim 0.3 \mathrm{pc}$, smaller than the sizes derived for clouds in the highly disturbed ISM of M82 by Lord et al. (1996) (0.4-1.0 pc), while the CO-emitting cores of these clouds must have size $<0.1 \mathrm{pc}$.

Yet another possible explanation for the lack of $\mathrm{CO}$ in 3C 368 is that the molecular clouds in the galaxy may be chemically young. Glover \& Clark (2012) have shown that $\mathrm{C}^{+}$ recombines with free electrons to form $\mathrm{C}^{0}$ in less than $\sim 0.1 \mathrm{Myr}$, while the subsequent formation of $\mathrm{CO}$ from the neutral atoms can take significantly longer, 1-3 Myr. It could be that we are observing these molecular clouds before they have had the chance to form an appreciable amount of $\mathrm{CO}$.

The work of Stoerzer et al. (1997) suggests that a chemically young ISM may be possible here. Cloudlets within a clumpy ISM, moving relative to one other, can periodically shield each other from the intense UV radiation field present in the ISM. In this scenario, the [C II] we observe would originate from the surfaces of dense cloudlets exposed to the intense UV field. The CO-emitting cores of the cloudlets would retreat toward the center each time they were exposed to the full UV field, and then take an appreciable time to reform after the next shielding event. This effect would be particularly pronounced if the cloudlets were small, and hence much of their volume was penetrated by UV photons between shielding events, as seems to be the case here. A testable prediction of this model would be the existence of considerable [C I] emission, since $\mathrm{C}^{+}$ recombines quickly after the shielding event is initiated. For this reason, we plan follow-up observations of 3C 368 in [C I] to try and resolve the mystery of the missing $\mathrm{CO}$.

\section{Future Observations}

In an effort to answer some of the remaining questions pertaining to $3 \mathrm{C} 368$, we have been awarded ALMA time to observe the [C II] $158 \mu \mathrm{m}$ line, at 0 !! 2 spatial resolution, to determine the extent of star formation in this source. The models we have employed here predict star formation extended over kilo-parsec scales, which, if correct, will appear in the ALMA observations as an extended source over $\sim 10$ beams.

In addition to the ionized carbon line, we have also been awarded time to observe the [C I] $609 \mu \mathrm{m}$ line in $3 \mathrm{C} 368$ (the $370 \mu \mathrm{m}$ line is blocked by the atmosphere). We expect that if the molecular clouds in the galaxy are indeed chemically young, the neutral carbon line will be very bright when compared to our $\mathrm{CO}$ line upper limit, since the $\mathrm{CO}$ would not have had sufficient time to form.

\section{Conclusions}

We have observed the fine-structure lines constituting the "oxygen toolkit" in 3C 368, including the [O I] $63 \mu \mathrm{m}$, [O III]
$52 \mu \mathrm{m}$ and $88 \mu \mathrm{m}$, and [O IV] $26 \mu \mathrm{m}$ lines. Using these oxygen lines together with archival fine-structure neon lines in the midinfrared, we have modeled the $\mathrm{H}$ II regions in 3C 368, using the models of Rubin (1985), and found them to be ionized by starbursts headed by 08 stars, with an age of $\sim 6.5 \mathrm{Myr}$. This age is comparable to the estimated age of the latest episode of AGN activity in 3C 368, determined by the propagation of the radio lobes.

Given the rate of star formation that we are witnessing in 3C 368, with the alignment of the optical and radio axes, and estimated age of both the starburst and most recent epoch of AGN activity, this source is a strong candidate for further study in the field of galaxy-BH coevolution.

We have also modeled the PDRs in 3C 368 and found them to be consistent with star formation spread over kilo-parsec distance scales. However, we did not detect any CO in 3C 368. Our $3 \sigma$ limit for the $\mathrm{CO}(2-1)$ line is $<0.201 \mathrm{Jy} \mathrm{km} \mathrm{s}^{-1}$, or equivalently $<7.3 \times 10^{-22} \mathrm{~W} \mathrm{~m}^{-2}$, a level 12 times smaller than the value expected from standard PDR models. The lack of $\mathrm{CO}$ emission may be due to a low-metallicity, highly fractionated, or possibly chemically young ISM. We find that the most likely explanation is that the ISM is highly fractionated into small clouds, perhaps as a result of the interactions between AGN winds and the star-forming molecular clouds, causing it to appear chemically young. Future ALMA observations will enable us to test this theory of large-scale star formation in the highly fractionated ISM of $3 \mathrm{C} 368$.

We thank the anonymous referee for the insightful comments and suggestions that helped to improve this manuscript. We additionally thank T. K. Daisy Leung and A. Gowardhan for help with preparing this manuscript in LaTeX. ZEUS observations were supported by NSF grant AST-01109476. C.L. acknowledges support from an NRAO Student Support Award, SOSPA3-011, and a New York Space Grant Award. D.B. acknowledges partial support from ALMA-CONICYT FUND No. 31140010. D.R. acknowledges support from the National Science Foundation under grant number AST-1614213 to Cornell University.

This paper makes use of the following ALMA data: ADS/JAO. ALMA\#2012.1.00426.S and ADS/JAO.ALMA\#2013.1.01223. S. ALMA is a partnership of ESO (representing its member states), NSF (USA) and NINS (Japan), together with NRC (Canada) and NSC and ASIAA (Taiwan) and KASI (Republic of Korea), in cooperation with the Republic of Chile. The Joint ALMA Observatory is operated by ESO, AUI/NRAO and NAOJ.

This work is based in part on observations made with the Spitzer Space Telescope, which is operated by the Jet Propulsion Laboratory, California Institute of Technology under a contract with NASA.

\section{References}

Archibald, E. N., Dunlop, J. S., Hughes, D. H., et al. 2001, MNRAS, 323, 417 Asplund, M., Grevesse, N., Sauval, A. J., \& Scott, P. 2009, ARA\&A, 47, 481 Bernard Salas, J., Pottasch, S. R., Beintema, D. A., \& Wesselius, P. R. 2001, A\&A, 367, 949

Best, P. N., Carilli, C. L., Garrington, S. T., Longair, M. S., \& Rottgering, H. J. A. 1998a, MNRAS, 299, 357

Best, P. N., Longair, M. S., \& Roettgering, H. J. A. 1998b, MNRAS, 295, 549

Best, P. N., Röttgering, H. J. A., \& Longair, M. S. 2000, MNRAS, 311, 1

Bolatto, A. D., Wolfire, M., \& Leroy, A. K. 2013, ARA\&A, 51, 207

Boreiko, R. T., Betz, A. L., \& Zmuidzinas, J. 1988, ApJL, 325, L47

Carilli, C. L., \& Walter, F. 2013, ARA\&A, 51, 105 
Chambers, K. C., Miley, G. K., \& Joyce, R. R. 1988, ApJL, 329, L75

Cormier, D., Lebouteiller, V., Madden, S. C., et al. 2012, A\&A, 548, A20

Crawford, C. S., \& Fabian, A. C. 1995, MNRAS, 273, 827

Croft, S., van Breugel, W., de Vries, W., et al. 2006, ApJ, 647, 1040

Dey, A., van Breugel, W., Vacca, W. D., \& Antonucci, R. 1997, ApJ, 490, 698

Djorgovski, S., Spinrad, H., Pedelty, J., Rudnick, L., \& Stockton, A. 1987, AJ, 93, 1307

Edge, D. O., Shakeshaft, J. R., McAdam, W. B., Baldwin, J. E., \& Archer, S. 1959, MmRAS, 68, 37

Emonts, B. H. C., Norris, R. P., Feain, I., et al. 2014, MNRAS, 438, 2898

Evans, A. S., Sanders, D. B., Mazzarella, J. M., et al. 1996, ApJ, 457, 658

Ferkinhoff, C., Hailey-Dunsheath, S., Nikola, T., et al. 2010, ApJL, 714, L147

Ferrarese, L., \& Merritt, D. 2000, ApJL, 539, L9

Gaibler, V., Khochfar, S., Krause, M., \& Silk, J. 2012, MNRAS, 425, 438

Genzel, R., Tacconi, L. J., Lutz, D., et al. 2015, ApJ, 800, 20

Glover, S. C. O., \& Clark, P. C. 2012, MNRAS, 421, 116

Gorjian, V., Cleary, K., Werner, M. W., \& Lawrence, C. R. 2007, ApJL, 655, L73

Hailey-Dunsheath, S., Nikola, T., Stacey, G. J., et al. 2010, ApJL, 714, L162

Hammer, F., Proust, D., \& Le Fevre, O. 1991, ApJ, 374, 91

Hernán-Caballero, A., Spoon, H. W. W., Lebouteiller, V., Rupke, D. S. N., \& Barry, D. P. 2016, MNRAS, 455, 1796

Hollenbach, D. J., \& Tielens, A. G. G. M. 1999, RvMP, 71, 173

Hönig, S. F., \& Kishimoto, M. 2010, A\&A, 523, A27

Houck, J. R., Roellig, T. L., van Cleve, J., et al. 2004, ApJS, 154, 18

Jackson, N., \& Rawlings, S. 1997, MNRAS, 286, 241

Kaufman, M. J., Wolfire, M. G., \& Hollenbach, D. J. 2006, ApJ, 644, 283

Kaufman, M. J., Wolfire, M. G., Hollenbach, D. J., \& Luhman, M. L. 1999, ApJ, 527, 795

King, A., \& Pounds, K. 2015, ARA\&A, 53, 115

Lebouteiller, V., Barry, D. J., Goes, C., et al. 2015, ApJS, 218, 21

Lebouteiller, V., Barry, D. J., Spoon, H. W. W., et al. 2011, ApJS, 196, 8

Lord, S. D., Hollenbach, D. J., Haas, M. R., et al. 1996, ApJ, 465, 703

Luhman, M. L., Satyapal, S., Fischer, J., et al. 1998, ApJL, 504, L11
Madden, S. C., Poglitsch, A., Geis, N., Stacey, G. J., \& Townes, C. H. 1997, ApJ, 483, 200

Maloney, P., \& Black, J. H. 1988, ApJ, 325, 389

McCarthy, P. J., van Breugel, W., Spinrad, H., \& Djorgovski, S. 1987, ApJL, 321, L29

Meijerink, R., Spaans, M., \& Israel, F. P. 2007, A\&A, 461, 793

Meisenheimer, K., \& Hippelein, H. 1992, A\&A, 264, 455

Meynet, G., \& Maeder, A. 2003, A\&A, 404, 975

Oberst, T. E., Parshley, S. C., Stacey, G. J., et al. 2006, ApJL, 652, L125

Ossenkopf, V., Röllig, M., Neufeld, D. A., et al. 2013, A\&A, 550, A57

Ott, S. 2010, in ASP Conf. Ser. 434, Astronomical Data Analysis Software and Systems XIX, ed. Y. Mizumoto, K.-I. Morita, \& M. Ohishi (San Francisco, CA: ASP), 139

Palay, E., Nahar, S. N., Pradhan, A. K., \& Eissner, W. 2012, MNRAS, 423, L35

Pilbratt, G. L., Riedinger, J. R., Passvogel, T., et al. 2010, A\&A, 518, L1

Podigachoski, P., Barthel, P. D., Haas, M., et al. 2015, A\&A, 575, A80

Poglitsch, A., Krabbe, A., Madden, S. C., et al. 1995, ApJ, 454, 293

Poglitsch, A., Waelkens, C., Geis, N., et al. 2010, A\&A, 518, L2

Rubin, R. H. 1985, ApJS, 57, 349

Rubin, R. H., Simpson, J. P., Colgan, S. W. J., et al. 2016, MNRAS, 459, 1875

Sanders, D. B., Soifer, B. T., Elias, J. H., et al. 1988, ApJ, 325, 74

Scoville, N., Sheth, K., Aussel, H., et al. 2016, ApJ, 820, 83

Silk, J. 2013, ApJ, 772, 112

Solomon, P. M., \& Vanden Bout, P. A. 2005, ARA\&A, 43, 677

Spergel, D. N., Verde, L., Peiris, H. V., et al. 2003, ApJS, 148, 175

Stacey, G. J., Charmandaris, V., Boulanger, F., et al. 2010a, ApJ, 721, 59

Stacey, G. J., Geis, N., Genzel, R., et al. 1991, ApJ, 373, 423

Stacey, G. J., Hailey-Dunsheath, S., Ferkinhoff, C., et al. 2010b, ApJ, 724, 957

Stacey, G. J., Smyers, S. D., Kurtz, N. T., \& Harwit, M. 1983, ApJL, 265, L7

Stoerzer, H., Stutzki, J., \& Sternberg, A. 1997, A\&A, 323, L13

Tielens, A. G. G. M., \& Hollenbach, D. 1985, ApJ, 291, 722

Vacca, W. D., Garmany, C. D., \& Shull, J. M. 1996, ApJ, 460, 914

Wolfire, M. G., Tielens, A. G. G. M., \& Hollenbach, D. 1990, ApJ, 358, 116 